\title{
Awareness of Human Papillomavirus Vaccine Among Adolescent African American Males Who Have Sex with Males: a Pilot Study
}

\author{
Onyekachi S. Onyeabor • Nicolle Martin • Verner N. Orish • \\ Adekunle O. Sanyaolu • Nnaemeka C. Iriemenam
}

Received: 27 August 2014 / Revised: 6 October 2014 / Accepted: 24 October 2014 / Published online: 4 December 2014

(C) W. Montague Cobb-NMA Health Institute 2014

\begin{abstract}
African American adolescent males who have sex with males (MSMs) have a high prevalence of sexually transmitted diseases (STDs) that has been directly linked to lack of access to primary care providers and reluctance to disclose their sexuality. The human papillomavirus (HPV) is the most common STD with more than 40 different serotypes and can lead to anal/genital warts as well as oral and genital cancers. The HPV vaccine if taken prior to an adolescent becoming sexually active serves a prophylactic function. The HPV vaccine is approved by the Food and Drug Administration (FDA) for girls and boys; however, HPV vaccination rates among adolescents within different minority and underserved communities have been disappointing even though these groups are disproportionately infected with the HPV virus and certain male-specific cancers. Little is known about the uptake of the vaccine among African American MSMs and thus the aim of this study. This qualitative study is based on the health belief model and assessed participants' level of awareness of HPV,
\end{abstract}

O. S. Onyeabor $(\bowtie)$

Satcher Health Leadership Institute, Department of Community

Health and Preventive Medicine, Morehouse School of Medicine,

720 Westview Drive, Atlanta, GA 30310, USA

e-mail: sonyeabor@msm.edu

\section{N. Martin}

Department of Community Health and Preventive Medicine, Morehouse School of Medicine, 720 Westview drive, Atlanta, GA 30310, USA

V. N. Orish

Department of Internal Medicine, Effia-Nkwanta Regional Hospital Sekondi-Takoradi, Sekondi-Takoradi, Western Region, Ghana

\section{A. O. Sanyaolu $\cdot$ N. C. Iriemenam}

Department of Medical Microbiology and Parasitology, College of

Medicine, University of Lagos, Idi-araba, Lagos, Nigeria

A. O. Sanyaolu

Department of Medical Microbiology and Immunology, Saint James

School of Medicine, Anguilla, British West Indies the HPV vaccine, and HPV-related illnesses among 24 African American male adolescents between 16 and 18 years old who self identify as MSMs. As part of a larger study, two focus groups were conducted for African American MSMs. Participants failed to understand their potential risk for HPV given the higher rates of STD infection experienced by MSMs. They expressed very little knowledge of the HPV vaccine and are also not aware of the complications of HPV virus infection. However, they were very eager to know more about the virus and the vaccine. This study demonstrates the need for the development of health communication intervention and more research targeting African American MSMs and also the need for policy change towards making the HPV vaccine routine for males especially adolescents at no cost.

Keywords Human papillomavirus vaccine $\cdot$ African American · Adolescent · Male

\section{Introduction}

Adolescent African American males who have sex with males (AAAMSMs) are vulnerable to sexually transmitted diseases (STDs) due to stigma, lack of social support, and poor access to primary care providers [1]. Lack of consistent partners, substance abuse, risky sexual behaviors, and lack of awareness of infection status have also been identified as some factors that could also predispose AAAMSMs to high prevalence of STDs. AAAMSMs have been identified as the most vulnerable population within the males who have sex with male (MSM) community in the USA [2]. The human papillomavirus (HPV) is a sexually transmitted virus which initially presents with no obvious symptoms while in the human host but might progress to cause cancers and other conditions depending on the part of the body where it is found. In the USA, there are about 6.2 million new HPV infections each 
year among people between 14 and 44 years; of these, $70 \%$ occur among those between 15 and 24 years [3]. Cervical cancer in women is one of the common gynecological cancers caused by HPV serotypes. Other conditions that can arise from HPV infection include anal, oral, and neck cancers as well as genital warts. Anal cancer, though a rare disease, is caused by HPV and associated with men who have sex with men especially when their body immunity has been weakened by other conditions like human immunodeficiency virus (HIV) infection, substance abuse, or smoking [4-6]. HPV infection and its complications can be prevented by having HPV vaccine before contracting the infection. The US Food and Drug Administration (FDA) approved two quadrivalent vaccines (HPV4) for HPV infection prevention: Gardasil and Cervarix. Both vaccines prevent infection with high-risk HPV strains, which cause nearly $70 \%$ of cervical cancers. In 2011, Gardasil HPV4 was approved for use in males. The Advisory Committee on Immunization Practices (ACIP) provided guidance that HPV4 may be given to males aged 11 through 21 years who have not been vaccinated previously or who have not completed a three-dose series to reduce their likelihood of acquiring genital warts and HPV-associated neoplasia. The HPV vaccine is also recommended for males of ages 22 through 26 especially for men who have sex with men and those whose immune system is weakened by HIV, other diseases, or drugs. The ACIP recommends routine vaccination of females at age 11 or 12 years and catch-up vaccination for females aged 13 through 26 years [7].

This study was aimed at understanding the level of awareness of HPV, the HPV vaccine, and HPV-related illness among AAAMSMs with the intent of understanding the need for future awareness creation and hopeful increase in uptake for the vaccine among this population with a higher risk for acquiring genital warts and other HPV-related cancers.

\section{Methods}

This study was approved by the Morehouse School of Medicine Institutional Review Board. The health belief model was used to assess participants' level of awareness of HPV, the HPV vaccine, and HPV-related illness among African American male adolescents who self identify as MSMs. As part of a larger study, two focus groups of 12 participants each were conducted for AAAMSMs. They were all between 16 and 18 years of age. Participants were selected by a snowballing sampling method through our collaboration with a local community-based organization, focusing only on participants who are either temporarily or permanently separated from their families and are able to take decision regarding participating in a study without the need to consult another person. All participants completed consent documents and understood the requirements and expectation for the study.
They were also compensated for their time. After the discussions, the recorded information were transcribed on paper by a paid consultant to the research team. The transcribed data and the notes taken during the focus group meeting were stored in a locked cabinet within the research office. We used NVivo 9 software for qualitative data analysis. Transcribed data, field notes, and memos were uploaded into the NVivo 9 software for coding, node formation, and theme selection. We ended up with 4 themes from 16 codes.

\section{Focus Groups}

Each focus group discussion lasted $90 \mathrm{~min}$ and was conducted by a paid trained focus group facilitator. All participants self identify as adolescent African American males who have sex with males and gave their consent for the study. They were informed that the discussion will be recorded, stored, and used for research only and that their identity will remain confidential. The recording equipment was also provided and tested prior to this time for clarity of recorded information. Participants were asked questions in line with the health belief model about HPV, HPV vaccine, and HPV disease complications. Discussion areas include (a) perceived susceptibility to HPV infection, (b) perceived severity of HPV infection, (c) perceived benefits of HPV vaccination, (d) perceived barriers to HPV vaccination, and (e) cues to action for HPV vaccination. Examples of questions for discussion are what do you know about human papillomavirus? Have you ever heard of human papillomavirus vaccine? During each of the focus group, notes were taken by a member of the research team.

\section{Codes, Categories, and Themes}

The coding process by two researchers using the NVivo 9 software involved choosing words, phrases, or groups of words from research data including the transcript that captured a portion of the transcript [8]. Once the codes were completed and compared between the researchers, 16 codes were selected and the categorization process allowed the codes to be segregated, grouped, or regrouped to consolidate meaning or explanation [9]. On completion of categorization process, four categories emerged. The four categories were further reorganized and discussed and finally we ended up with four themes (Fig. 1).

\section{Results}

Our results indicated that $75 \%$ of the participants were aware of the HPV vaccine for girls, while $100 \%$ of them were unaware of a HPV vaccine for males (Table 1). However, 
Fig. 1 Transcribed data was sorted into codes, categories, and then themes
- HPV infection

- Sexually active

- Anybody can get it

- Protected sex

- Anal cancer

- HPV information

- Women cancer

- Young teenage girls

- Black gay men

- Prevention

- Romantic relationships

- Information search

- Doctors

- Healthcare decision

- WebMD

- $\quad$ Face to face meeting
Categories

1. HPV infection, HPV vaccine and disease complications

2. Predisposing factors to HPV infection

3. Information resources on HPV, HPV Vaccine

4. Misconceptions around HPV Vaccine

Themes
1. HPV infection and
complications
2. HPV Vaccine
3. Barriers to HPV vaccine
access
4. Preventive intervention

the participants were very receptive to the idea of a preventive vaccine against HPV even though $83.3 \%$ of the participants lacked the knowledge of the natural history of HPV infection and complications. They were very reluctant about the vaccination since it is a new vaccine for males. Most of the participants would rather wait for more research and awareness to be created before they could accept to be vaccinated mainly because of fear of potential side effects of the vaccine in males. They were also very concerned about the cost of receiving HPV vaccine, alluding if health insurance would

Table 1 Self-reported awareness of HPV vaccine

\begin{tabular}{|c|c|c|}
\hline Awareness & $\begin{array}{l}\text { No } \\
N(\%)\end{array}$ & $\begin{array}{l}\text { Yes } \\
N(\%)\end{array}$ \\
\hline Participants are aware of HPV vaccine for males & $24(100)$ & $0(0)$ \\
\hline Participants are aware of HPV vaccine for girls & $6(25)$ & $18(75)$ \\
\hline Participants have been vaccinated against HPV & $24(100)$ & $0(0)$ \\
\hline $\begin{array}{l}\text { Participants are aware of the complications of HPV } \\
\text { infection }\end{array}$ & $20(83.3)$ & $4(16.7)$ \\
\hline $\begin{array}{l}\text { Participants feel that they are predisposed to HPV } \\
\text { because of their sexuality }\end{array}$ & $16(66.7)$ & $8(33.3)$ \\
\hline
\end{tabular}

$N$ number of participants cover the cost. Interestingly, $66.7 \%$ of participants do not see themselves as more predisposed to HPV just because they are males who have sex with males.

\section{Discussion}

Since the approval of the HPV vaccine by FDA in the USA for use in males, adolescents and young adult males still do not understand why they should receive HPV vaccine. Adolescent African American males who have sex with males are even more predisposed to sexually transmitted infections and are less likely to have a primary care physician who could talk to them about it. This study used the health belief model to understand the experience of some adolescent African American males who have sex with males regarding HPV and HPV vaccine. Generally, participants were favorable to vaccination especially for the diseases they are already aware of, but they were totally ( $100 \%$ ) unaware of the approval of the HPV vaccine for use in males. They thought it is meant for only young girls for prevention of cervical cancer. They expressed reluctance in receiving HPV vaccination since possible complications in males are not known, so they would 
rather wait for some years or until they are well informed about the HPV vaccine in males. They were also uncomfortable with the fact that it might cost some money to get the vaccine which is the case when vaccination is not freely available especially in developing and even some developed countries that have not adopted HPV vaccination for males as a routine vaccine.

HPV infection is the commonest sexually transmitted infection in the USA and the cause of cervical cancer with the highest rate of infection observed among individuals aged 1527 [3]. The estimated prevalence of HPV infection among asymptomatic males aged 18-70 was $65.2 \%$ [10], although most of the infection does not lead to severe complications like anal, penile, or oropharyngeal cancers. An estimated $63 \%$ of oropharyngeal cancers, $93 \%$ of anal cancers, and $36 \%$ of penile cancers in the USA are attributable to infection with oncogenic HPV types (mainly types 16 and 18) [11]. Nononcogenic HPV types (types 6 and 11) cause anogenital warts [12]. About $83.3 \%$ of the participants in this study had no knowledge of the link between anal cancer and HPV infection. They also failed to appreciate their potential risk of being infected with HPV because of their sexuality and also because most of them do not have primary care physician and even when they do, they are often not comfortable with disclosing their sexuality because of the stigma. Lack of awareness of HPV is common in literature especially in developing countries and underserved communities [13], and that as well as affordability drives poor uptake of the HPV vaccine in these communities. Good knowledge or increased awareness of HPV infection will greatly affect how receptive this vulnerable population will be to receiving HPV vaccine. Affordable Care Act (ACA) presents a wonderful opportunity for these adolescents to have access to primary care physicians for an increased possibility for more awareness and knowledge about HPV infections and complications.

Evidence shows that awareness and affordability of the HPV vaccine are the reasons for reduced access of the vaccine to both female and male populations [14], and because the HPV vaccine for males is not yet a routine vaccine in some areas, it might come at a cost which may not be affordable to adolescent African American males who have sex with males. However, study participants, apart from having similar barriers to vaccine access, are also disadvantaged because they are African American adolescent males who have sex with males [15] and because their sexuality is not known to their families due to fear of being rejected by family and the community. Around $66.7 \%$ of these participants exhibited a level of ignorance about their vulnerability to sexually transmitted infections (STIs) even though young African American men who have sex with men have the highest prevalence of STIs including HPV and HIV in the USA [16]. An intervention program targeting young African American males who have sex with males will be very essential to help create more awareness about safe sexual behavior and the need to take advantage of a preventive measure like the HPV vaccine to reduce the possibility of male-related sexual cancers that are prevalent in men who have sex with men [17]. Participants were very receptive of preventive intervention measures like the HPV vaccine. They were worried about possible complications from the HPV vaccine since the vaccine is mainly used for young females against cervical cancer. They would rather wait for more information about the HPV vaccine before accepting it. This is similar to the findings by other studies with regard to HPV acceptability among gays [14]. However, participants do not perceive themselves as more vulnerable to sexually transmitted infections like HPV than other people. They also have no idea about their predisposition to anal cancers as a result of HPV infection. There were few limitations to this study. We had only 24 African American adolescents who participated in two focus group discussions. It would be more informative to also have adolescent males who have sex with males from other races. However, participants' views during this study cannot be generalized especially because of the small number of participants as well as the lack of objective data.

\section{Conclusion}

There is high level of ignorance about HPV infection and complications as well as the HPV vaccine for males among this population of adolescent African American males who have sex with males. But, they were very open to the idea of a preventive vaccine against a sexually transmitted infection and would only like to be vaccinated if the vaccine is free and more knowledge about side effects of the vaccine in males is known. They do not see their sexuality as being vulnerable to HPV infection transmission. More research about HPV vaccine uptake targeting larger populations of young MSMs from underserved populations is needed to create more awareness and also provide more data for policy making especially towards reducing the high prevalence of HPV-associated cancers among this vulnerable population in the USA.

Acknowledgments The authors express their gratitude to all the participants for their immense contributions and support.

Conflict of Interest Authors Onyeabor, Martin, Orish, Sanyaolu, and Iriemenam declare that they have no conflict of interest.

Ethics All procedures followed were in accordance with the ethical standards of the responsible committee on human experimentation (institutional and national) and with the Helsinki Declaration of 1975, as revised in 2000. Informed consent was obtained from all patients for being included in the study. 


\section{References}

1. Dorell CG, Sutton MY, Oster AM, Hardnett F, Thomas PE, Gaul ZJ, et al. Missed opportunities for HIV testing in health care settings among young African American men who have sex with men: implications for the HIV epidemic. AIDS Patient Care STDS. 2011;25:657-64.

2. Finlayson TJ, Le B, Smith A, Bowles K, Cribbin M, Miles I, et al. HIV risk, prevention, and testing behaviors among men who have sex with men-National HIV Behavioral Surveillance System, 21 U.S. cities, United States, 2008. MMWR Surveill Summ. 2011;60:1-34.

3. Weinstock H, Berman S, Cates Jr W. Sexually transmitted diseases among American youth: incidence and prevalence estimates, 2000. Perspect Sex Reprod Health. 2004;36:6-10.

4. Palefsky J. Human papillomavirus and anal neoplasia. Curr HIV/ AIDS Rep. 2008;5:78-85.

5. Skaaby S, Kofoed K. Anogenital warts in Danish men who have sex with men. Int J STD AIDS. 2011;22:214-7.

6. Simatherai D, Bradshaw CS, Fairley CK, Bush M, Heley S, Chen MY. What men who have sex with men think about the human papillomavirus vaccine. Sex Transm Infect. 2009;85:148-9.

7. CDC. Quadrivalent human papillomavirus vaccine. Recommendations of the Advisory Committee on Immunization Practices (ACIP) MMWR. 2007;56:1-24.

8. Saldana J. The coding manual for qualitative researchers. SAGE Publications. 2009.

9. Kitto SC, Chesters J, Grbich C. Quality in qualitative research. Med J Aust. 2008;188:243-6.
10. Giuliano AR, Anic G, Nyitray AG. Epidemiology and pathology of HPV disease in males. Gynecol Oncol. 2010;117:S159.

11. Gillison ML, Chaturvedi AK, Lowy DR. HPV prophylactic vaccines and the potential prevention of noncervical cancers in both men and women. Cancer. 2008;113:3036-46.

12. Greer CE, Wheeler CM, Ladner MB, Beutner K, Coyne MY, Liang $\mathrm{H}$, et al. Human papillomavirus (HPV) type distribution and serological response to HPV type 6 virus-like particles in patients with genital warts. J Clin Microbiol. 1995;33:2058-63.

13. Wang Z, Mo PK, Lau JT, Lau M, Lai CH. Acceptability of HPV vaccines and perceptions related to genital warts and penile/anal cancers among men who have sex with men in Hong Kong. Vaccine. 2013;31:4675-81.

14. Lau JT, Wang Z, Kim JH, Lau M, Lai CH, Mo PK. Acceptability of HPV vaccines and associations with perceptions related to HPV and HPV vaccines among men who have sex with men in Hong Kong. PLoS One. 2013;8:e57204.

15. Gelman A, Miller E, Schwarz EB, Akers AY, Jeong K, Borrero S. Racial disparities in human papillomavirus vaccination: does access matter? J Adolesc Health. 2013;53:756-62.

16. Prejean J, Song R, Hernandez A, Ziebell R, Green T, Walker F, et al. Estimated HIV incidence in the United States, 2006-2009. PLoS One. 2011;6:e17502.

17. Colon-Lopez V, Ortiz AP, Del Toro-Mejias L, Clatts MC, Palefsky JM. Epidemiology of anal HPV infection in high-risk men attending a sexually transmitted infection clinic in Puerto Rico. PLoS One. 2014;9:e83209. 